\title{
USO INDISCRIMINADO DE METILFENIDATO E LISDEXANFETAMINA POR ESTUDANTES UNIVERSITÁRIOS PARA APERFEIÇOAMENTO COGNITIVO
}

\author{
Nadinni Silva Vilas Boas Cerqueira ${ }^{1}$ \\ Bruna do Carmo Almeida ${ }^{2}$ \\ Raineldes Avelino Cruz Junior ${ }^{3}$
}

\begin{abstract}
RESUMO: Observando os aspectos legais, e subjetivando os medicamentos que se sujeitam ao controle da portaria $n^{\circ} 344 / 98$, legislação que dispõe sobre medicamentos e substâncias que estão sujeitas a controle especial. Metilfenidato e lisdexanfetamina são substâncias controladas que pertencem à lista $\mathrm{A}_{3}$ da portaria. Ambas são prescritas para o tratamento de TDAH (Transtorno de Déficit de Atenção e Hiperatividade). No qual é definida como uma condição do neurodesenvolvimento, e apresenta os seguintes sintomas: desatenção, hiperatividade e/ou impulsividade. A farmacoterapia utilizada para o tratamento de TDAH, é o cloridrato de metilfenidato conhecido comercialmente por Ritalina, ou Concerta e o dimesilato de lisdexanfetamina, conhecido comercialmente por Venvanse. Porém, é crescente o uso dessas substâncias sem receita médica entre estudantes universitários saudáveis, com intuito de potencializar o aprimorando cognitivo, principalmente em períodos de estudos e de grande estresse. Este Artigo tem como objetivo, avaliar o princípio ativo, mecanismo de ação e frequência de uso e os possíveis riscos da utilização inadequada do metilfenidado e da lisdexanfetamina por estudantes universitários para o aprimoramento cognitivo. Este artigo trata-se de uma revisão bibliográfica narrativa, escrita a partir de trabalhos científicos divulgados nas bases de dados: Scientific Eletronic Library Online (SciELO), PePSIC da Biblioteca Virtual em Saúde (BVS) e PUBMED. Nos idiomas, português e inglês com restrição do tempo de publicação no período de 2012 a 2021. Foram analisados quatro artigos referentes ao tema uso não prescrito de metilfenidato e/ou lisdexanfetamina por estudantes universitários. Através dos presentes artigos estudados, alcançamos grandes resultados de uma evidente relação ao uso indiscriminado do metlfenidado e da lisdexanfetamina por estudantes universitários para aprimoramento cognitivo. Podendo ressaltar a grande importância e relevância do uso racional de medicamentos.
\end{abstract}

Palavras-chave: Metilfenidato and/or Lisdexanfetamina. TDAH. Uso não prescrito de psicoestimulantes.

ABSTRACT: Observing the legal aspects, and subjecting the drugs that are subject to the control of Ordinance No. 344/98, legislation that provides for drugs and substances that are subject to special control. Methylphenidate and lisdexamphetamine are controlled substances that belong to the $\mathrm{A}_{3}$ list of the ordinance. Both are prescribed for the treatment

\footnotetext{
I Graduanda em Farmácia. Instituição: Universidade Salvador (UNIFACS). E-mail: nadyvb@hotmail.com Graduanda em Farmácia. Instituição: Universidade Salvador (UNIFACS)E-mail: bruna.almeida357@hotmail.com

3 Orientador. Mestre em Química Analítica pela Universidade Federal da Bahia - UFB. Docente da Universidade Salvador (UNIFACS). E-mail: raineldes.junior@unifacs.br
} 
of ADHD (Attention Deficit Hyperactivity Disorder). In which it is defined as a neurodevelopmental condition, and presents the following symptoms: inattention, hyperactivity and/or impulsivity. The pharmacotherapy used for the treatment of ADHD is methylphenidate hydrochloride, commercially known as Ritalin, or Concerta, and lisdexamphetamine dimesylate, commercially known as Venvanse. However, the use of these substances without a prescription among healthy university students is increasing, with the aim of enhancing cognitive improvement, especially during periods of study and high stress. This article aims to evaluate the active ingredient, mechanism of action and frequency of use and the possible risks of inappropriate use of methylphenidate and lisdexamphetamine by university students for cognitive improvement. This article is a narrative bibliographic review, written from scientific works published in the following databases: Scientific Electronic Library Online (SciELO), PePSIC from the Virtual Health Library (VHL) and PUBMED. In the languages, Portuguese and English, with restriction of publication time in the period from 2012 to 2021. Four articles referring to the topic nonprescription use of methylphenidate and/or lisdexamphetamine by university students were analyzed. Through the present articles studied, we have achieved great results from an evident relationship to the indiscriminate use of metlphenidate and lisdexamphetamine by university students for cognitive improvement. It can highlight the great importance and relevance of the rational use of medicines.

Keywords: Methylphenidate and/or Lisdexamphetamine. TDAH. Non-prescription use of psychostimulants.

\section{INTRODUÇÃO}

Observandos os aspectos legais, e subjetivando os medicamentos que se sujeitam ao controle da portaria $n^{\circ} 344 / 98$, legislação que dispõe sobre medicamentos e substâncias que estão sujeitas a controle especial. Metilfenidato e lisdexanfetamina são substâncias controladas que pertencem à lista $\mathrm{A}_{3}$ da portaria. Ambos são prescritos para o tratamento de TDAH (Transtorno de Déficit de Atenção e Hiperatividade).

De acordo com Castro et al. (2018) O Transtorno de Déficit de Atenção e Hiperatividade (TDAH), é definida como uma condição do neurodesenvolvimento. Apresenta sintomas como: desatenção, impulsividade e hiperatividade. A prevalência mundial do TDAH chega a ser estimada em 5,3\% aproximadamente. Costuma surgir com frequência na infância, mas pode persistir também na vida adulta, ocasionando prejuízo no desenvolvimento social, acadêmico e profissional.

A farmacoterapia mais utilizada para o tratamento dessa síndrome são os estimulantes do sistema nervoso central (SNC) cloridrato de metilfenidato, conhecido comercialmente por Ritalina ${ }^{\circledR}$, ou Concerta ${ }^{\circledR}$ e o dimesilato de lisdexanfetamina, conhecido comercialmente por Venvanse ${ }^{\circledR}$. (SILVA, 2020) 
As aplicações terapêuticas do metilfenidato são destinadas para: aumento da concentração, atenção, memória, e também para diminuição do sono. Em casos de narcolepsia (condição neurológica crônica no qual o indivíduo tem sono em excesso) existe uma estimulação do SNC, diminuição da fadiga, elevação no ânimo, e aumento da atenção.

A lisdexanfetamina é muito útil também no tratamento de pacientes em que houve falha terapêutica inicial com metilfenidato. Todavia, efeitos adversos como distúrbios do sono, perda de apetite e irritabilidade podem ser mais frequentes e menos tolerados por alguns indivíduos. (NETA et al. 2020).

É crescente o uso de metilfenidato e dimesilato de lisdexanfetamina sem receita médica entre estudantes universitários saudáveis, com intuito de potencializar o aprimoramento cognitivo, principalmente em períodos de estudos e de grande estresse. (ROCHA, 2016) Porém a utilização inadequada desses psicoestimulantes podem ocasionar grandes riscos para a saúde desses indivíduos. Portanto, o objetivo desse trabalho será avaliar o princípio ativo, mecanismo de ação, frequência de uso e os possíveis riscos da utilização inadequada do metilfenidato e da lisdexafetamina por estudantes universitários para aprimoramento cognitivo.

\section{DESENVOLVIMENTO}

\section{METILFENIDATO}

O metilfenidato é uma substância derivada da anfetamina, princípio ativo da Ritalina ${ }^{\circledR}$, medicamento produzido e comercializado pelo laboratório Novartis Biociências, e utilizada terapeuticamente no tratamento da Narcolepsia e TDAH. A substância também é comercializada sob nome comercial Concerta ${ }^{\circledR}$ produzida pelo laboratório Janssen Cilag, menos consumida no Brasil do que a Ritalina ${ }^{\circledR}$.

O metilfenidato surgiu no início da década de 40, como resultado da pesquisa de um fármaco que apresentasse menos efeitos adversos que a benzedrina, nome comercial da mistura racémica de anfetamina, lançada em 1933 pelo laboratório Smith, Kline and French. (COSTA, 2016)

Segundo Brant e Carvalho (2012) o metilfenidato foi sintetizado pelo antigo farmacêutico da empresa CIBA (atualmente, Novartis S/A) na Suíça, Leandro Panizzon, e foi patenteado em 1954, ou seja, garantiu assim a exclusividade ao explorar comercialmente a sua criação, passando a ser comercializado no mesmo ano e País como psicoestimulantes, 
medicamento no qual tem a função de contribuir para atividade motora e cognitiva. A chegada do metilfenidato no Brasil ocorreu por volta do ano de 1988. O nome Ritalina se deu através do apelido da esposa do Dr. Panizzon. Marguerite se transformou em Rita e, depois, em Ritaline.

Crianças com diagnóstico de TDAH devem ter pelo menos seis anos de idade ou mais antes de começarem a tomar este medicamento. (VERGHESE \& ABDIJADID, 202I) É um derivado da benzilpiperidina e fenetilamina cuja fórmula química é $\mathrm{C}_{14} \mathrm{H}_{19} \mathrm{NO}_{2}$ e o nome químico é metil 2-fenil- 2-(2-piperidil) acetato. Apresenta uma massa molar de 233,3II $\mathrm{g} / \mathrm{mol}$ e um tempo de semi-vida ( $\mathrm{t}^{1 / 2}$ ) entre as duas e três horas. (COSTA, 2016)

Segundo Verghese \& Abdijadid (202I) o metilfenidato tem como mecanismo de ação:

Bloquear a recaptação de dois neurotransmissores, norepinefrina (NE) e dopamina, pelos
neurônios pré-sinápticos. Mais especificamente, inibe os transportadores desses
neurotransmissores, aumentando a concentração de dopamina e norepinefrina na fenda
sináptica. Em geral, isso cria seu efeito estimulante clássico no sistema nervoso central
(SNC), principalmente no córtex pré-frontal. É quimicamente derivado da fenetilamina e
da benzilpiperazina. É metabolizado pelo fígado em ácido ritalínico por meio de um
processo denominado desesterificação via carboxilesterase CES IAI. Comparado a outros
medicamentos (ou seja, anfetaminas) derivados da fenetilamina, o metilfenidato parece
aumentar a taxa de ativação dos neurônios.

Em alguns estudos foram encontrados alguns efeitos colaterais relacionando ao uso do metilfenidato em curto prazo. Destacando: Dor de cabeça, nervosismo, ansiedade, insônia, sonolência, e aumento da frequência cardiaca .

De acordo com Monteiro et al. (2017) em uma análise realizada através de bases de dados do Instituto Federal Alemão ligado a medicamentos e dispositivos médicos, foi capaz de identificar entre 1999 a 201223 casos de dependências e abuso do uso de metilfenidato. 5 pessoas, dentre os 23 casos, relataram ter tido reações adversas como: transtorno de personalidade, depressão e a associação de ambos os sintomas.

\section{LISDEXANFETAMINA}

Segundo Neta et al. (2020) a Shire Pharmaceuticals em 26 de fevereiro de 2007, recebeu do Food and Drug Administration (FDA), Agência federal do Departamento de Saúde e Serviços Humanos dos Estados Unidos, que contribuem para o controle de alimentos e medicamentos para a saúde pública, foi aprovada o dimesilato de Lisdexanfetamina para o tratamento de TDAH, sendo comercializado nos Estados Unidos com o nome fantasia Vyvanse. A Agência Nacional de Vigilância Sanitária (ANVISA) 
concedeu em julho de 2010 a comercialização do medicamento no Brasil com o nome comercial Venvanse ${ }^{\circledR}$.

O dimesilato de lisdexanfetamina (LDX),é o primeiro pró-fármaco estimulante de longa ação para o tratamento do TDAH. (COGHILL, et al. 20I4.) ou seja, é um composto farmacologicamente inativo que ao sofrer biotransformação metabólica se torna um fármaco ativo.

No dizer de Neta et al. (2020), A lisdexanfetamina é um enantiômero único - (dextro) anfetamina - com fórmula molecular: $\mathrm{C}_{15} \mathrm{H}_{25} \mathrm{~N}_{3} \mathrm{O}$, massa molar de $263,37 \mathrm{~g} / \mathrm{mol}$.

Após a ingestão e absorção, o LDX é hidrolisado enzimaticamente para liberar a porção terapeuticamente ativa d-AMP e o aminoácido essencial lisina. (COGHILL, et al. 2014.)

Segundo Okamura (2019, p. 24) a lisdexanfetamina tem como mecanismo de ação:

A liberação de catecolaminas e a inibição da captura das mesmas. Desta maneira, acredita-se que após sua administração e hidrólise, ad-anfetamina liberada pela lisdexanfetamina (LDX), atue na liberação de norepinefrina e dopamina, na inibição da monoamina oxigenasse (MAO), dos transportadores de norepinefrina (NET), resultando assim numa ação estimulante do sistema nervoso central (SNC).

Takeda (202I, p. 2) descreve que: o uso abusivo da lisdexafetamina pode trazer grades riscos à saúde dos indivíduos que faz uso, podendo levar a dependência psicológica em diferentes graus.

\section{FREQUÊNCIA DE USO DOS PSICOESTIMULANTES}

A procura do aumento das oportunidades profissionais, na possibilidade de ganhar salários melhores e ser reconhecido profissionalmente, conquistar um diploma não é tão fácil. Para que tenha a garantia do sucesso é necessário abdicar de ações e estudar. Porém, para isso a capacidade de se concentrar é fundamental, mas, apesar de ser importante nem sempre é entendível. Logo, para evitar frustrações, alguns estudantes procuram por métodos que sejam capazes de proporcionar maiores rendimentos nos estudos. A potencialização do desempenho cognitivo que o fármaco apresenta chama atenção de universitários saudáveis para fazer uso do mesmo.

$\mathrm{Na}$ busca de melhorar o desempenho cognitivo, estudantes universitários em muitos países têm feito o consumo de psicoestimulantes, com a intenção de potencializar os resultados nas avaliações, originando dessa forma um comercio de substâncias dentro dos campos universitários. O uso de metilfenidato entre estudantes norte-americanos é 
estimado entre 5 a 7\%. Já no Brasil, também foi evidenciada esta prática, segundo um estudo realizado pelas representações sociais sobre o uso de metilfenidato para o aprimoramento cognitivo farmacológico.

\section{USO EXCESSIVO DAS SUBSTÂNCIAS}

Medicamentos psicoestimulantes possuem a capacidade de aumentar a motivação e o estado de alerta. Diminuindo a necessidade do descanso, Além de apresentar propriedades antidepressivas, proporcionando melhora no humor e no desempenho cognitivo. (NETO et al. 2018) O uso excessivo de anfetaminas pode desencadear sintomas de abstinência como: fadiga, depressão, insônia, irritabilidade, hiperatividade, labilidade emocional e psicose.

Os universitários que fazem uso de estimulantes do sistema nervoso central, sendo eles metilfenidado e a lisdexanfetamina, estão expostos a trazer grandes riscos para a sua saúde. Como caracteriza Neto et al. (2018) a utilização irracional de substâncias psicoativas para o aprimoramento cognitivo, podem causar dependência e danos ao organismo do usuário, principalmente se administrado de forma contínua e exagerada. Podendo causar sérias reações como: Alucinações, irritabilidade, ansiedade, cefaleia, tontura, taquicardia, náuseas, vômitos, diarreia, redução do apetite, perda de peso, dores abdominais, alterações hipofisárias e sexuais.

O uso em longo prazo do metilfenidato é capaz de originar um efeito denominado como dose-dependente, podendo desencadear insônia, depressão e exaustão vespertina, em casos de retirada brusca da medicação após um tempo maior de utilização dessa substância.

\section{ANÁLISE DE DADOS}

Reporta os seguintes dados: A primeira análise obtida através de uma coleta de dados realizada em setembro de 2014 por Cândido et al. (2019) na Universidade Federal de Minas Gerais (UFMG) com o objetivo de determinar a prevalência e os fatores associados ao uso de metilfenidato por universitarios brasileiros. Relata que, dos 378 alunos entrevistados, 67\% declararam ter de 20 a 30 anos; 5,8\% afirmaram ter feito uso de metilfenidato para neuroaprimoramento, sendo que $41 \%$ dos alunos, fizeram uso 4 semanas antes da pesquisa. Dentre os $5 \%$ dos estudantes que relatou fazer o uso do metilfenidato, $27 \%$ conseguiram adquirir o fármaco sem prescrição médica, nestes casos, o uso foi 
incentivado através de indicações de amigos. 62,5\% justificaram o uso da sua prescrição para TDAH (transtorno de déficit de atenção e hiperatividade). Na entrevista o uso "off label" Termo utilizado para descrever o uso não homologado de um determinado fármaco, estipulou que 9,1\% faz uso recreativo e 9,1\% para a diminuição do sono diurno.

Em contrapartida, um trabalho realizado por Affonso et al. (2016) com o intuito de identificar e quantificar o uso não terapêutico do cloridrato de metilfenidato, analisar os efeitos colaterais provocados por tal medicamento e fazer um levantamento do uso de outros psicoestimulantes pelos estudantes dos cursos de saúde da Faculdade Anhanguera de Brasília (FAB). Destacou-se, que o estudo envolveu 400 estudantes com idade maior que i8 anos. No período de 3 a 5 de novembro de 2014. 73\% do público feminino, dentre elas 40,9\% não possuía filho na faixa etária de 18 e 24 anos.

Analisando os resultados, percebeu-se que, 19,5\% dos estudantes relataram que fizeram uso de algum medicamento para auxiliar nos estudos; 48\% destes afirmaram ter utilizado o medicamento através de orientações de amigos; $9 \%$ indicação farmacêutica e 66\% sem prescrição médica. Os efeitos adversos que foram destacados pelos estudantes que faziam uso do metilfenidato foram: (cefaleia), taquicardia, insônia e boca seca. Apenas 13\% dos ouvintes, relataram que não sentiram nenhum tipo de efeito adverso. Dentre eles 39\% não faria uso do medicamento novamente. Segundo a pesquisa realizada na FAB $57 \%$ dos estudantes relatou que o principal motivo para o consumo do medicamento foi o aumento da concentração, $26 \%$ a diminuição do sono, e apenas $17 \%$ utilizou o metilfenidato para o tratamento do TDAH. A melhora na capacidade da concentração durante os estudos é confirmada pelos entrevistados, relatando também maior assimilação do conteúdo estudado.

Em uma pesquisa realizada por Pires et al. (2018) com objetivo de: determinar a utilização de medicamentos psicoestimulantes, sem necessidade médica, pelos estudantes de Medicina da Faculdade Governador Ozanam Coelho (FAGOC) de Ubá-MG. Determinou, que $99 \%$ faz uso de alguma substância psicoestimulantes, destacando o metilfenidato, no qual correspondeu a $56,56 \%$ da pesquisa; a maior parte do público participante é masculino $(68,42 \%) ; 76,76 \%$ relata fazer o uso sem prescrição médica; $66 \%$ começou a fazer uso após ingressar na faculdade; $88 \%$ faz uso em véspera de prova e $84,21 \%$ dos estudantes relataram o aumento da concentração com o uso das substâncias, conseguindo obter o resultado proposto do medicamento para o aprimoramento cognitivo. Dentre os entrevistados $52 \%$ dos alunos 
afirmaram que obteve melhora na concentração e $84,2 \%$ relataram ter seu rendimento acadêmico melhorado, $69,73 \%$ afirmaram apresentar efeitos colaterais, mas apenas 37,73\% suspenderam a medicação.

Com objetivo de determinar a prevalência e analisar os fatores associados ao uso indiscriminado de estimulantes do Sistema Nervoso Central entre estudantes brasileiros (acadêmicos e em preparação para concursos e vestibulares) como estratégia para melhorar o rendimento nos estudos. Wachholzr (2020) desenvolveu uma análise através de uma pesquisa quantitativa com 203 estudantes de concursos e vestibulares das cinco regiões nacionais (Sul, Sudeste, Norte, Nordeste e Centro-Oeste), os dados foram adquiridos por meio de um questionário criado na plataforma Google Forms, o questionário foi enviado através das redes sociais e ficou disponível na plataforma entre o dia 13 de novembro de 2019 ao dia 28 de fevereiro de 2020. 63\% do público que respondeu a pesquisa foi do gênero feminino, 35\% masculino, e 2\% preferiram não informar; 79\% dos estudantes alegaram fazer uso de estimulantes do sistema nervoso central, enquanto $21 \%$ responderam que já usou ou usa algum tipo de estimulante para o aprimoramento cognitivo. Dentre os estudantes que afirmaram já ter feito o uso, 63\% corresponde à área de biológicas/saúde, 21\% humanas, e I6\% exatas, $79 \%$ dos entrevistados relataram que se sentiam melhor ao fazer uso do medicamento, $19 \%$ responderam que o uso não interferia na sua produtividade e $2 \%$ não informaram. Os medicamentos citados pelos entrevistados foram: Ritalina ${ }^{\circledR}(48 \%)$, Venvanse ${ }^{\circledR}$ (14\%) e a Ritalina LA ${ }^{\circledR}$ (longa duração) (4\%), 32\% mencionaram outros estimulantes; $54 \%$ fizeram uso sem prescrição, $42 \%$ com prescrição, e $5 \%$ dos estudantes preferiram não informar.

\section{CONSIDERAÇÕES FINAIS}

Com o presente estudo, conclui-se que existe uma prevalência do uso de estimulantes do SNC, o metilfenidato e a lisdexanfetamina por estudantes universitários, com o intuito de potencializar a concentração nos estudos. Os principais motivos que levam os estudantes a fazerem o uso são: aumento da concentração, aprimoramento acadêmico e privação de sono. Visando obter um desempenho acadêmico positivo.

De acordo com os estudos disponíveis, torna-se evidente que o consumo indevido do metilfenidato e da lisdexanfetamina, pode se tornar um problema de saúde pública. 
Avaliando que os fármacos foram obtidos em alguns casos através de indicação e sem prescrição médica, aniquilando os efeitos colaterais que esses psicoestimulantes podem causar, podemos citar: cefaleia, taquicardia, boca seca e insônia.

Com base nestas considerações, sugere-se que haja controle na dispensação e prescrição dessas substâncias, uma fiscalização mais rigorosa nos medicamentos à base de lisdexanfetamina e metilfenidato. Como também a implementação de palestras em campos universitários abordando os perigos da automedicação, promovendo assim o uso racional desses medicamentos.

\section{REFERÊNCIAS}

AFFONSO, R. D. S. et al. O uso indiscriminado do cloridrato de metilfenidato como estimulante por estudantes da área da Saúde da Faculdade Anhanguera de Brasília (FAB). Infarma, Rio de Janeiro, v. 28, n. 3, p. 166-172, ago./2016. Disponível

$\mathrm{m}:$

http://revistas.cff.org.br/?journal=infarma\&page $=$ article\&op $=$ view\&path\% $\%$ B $\% 5 D=140$ 4\&path\%5B\%5 $\mathrm{D}=$ pdf. Acesso em: i5 nov. 2021.

BRANT, Luiz Carlos; CARVALHO, T. R. F. Metilfenidato: medicamento gadget da contemporaneidade. Scielo, Minas Gerais, v. 16, n. 42, p. 623-636, set./2012. Disponível

m:

https://www.scielo.br/j/icse/a/rHMChQ97YKsSs8JD3X8rVDt/?format=pdf\&lang=pt.

Acesso em: 26 out. 202I.

BRITO, Jéssica Raizi; CECATTO, Luis Humberto. Transtorno de Déficit de Atenção/ Hiperatividade (TDAH): Um olhar voltado para os pais. Pepsic, [S. 1.], v.52, n. 2, p. 67-79, dez./2019. Disponível em: http://pepsic.bvsalud.org/pdf/aletheia/v52n2/v52n2ao6.pdf. Acesso em: I4 nov. 2021.

CÂNDIDO, R. C. F. et al. Prevalência e fatores associados ao uso demetilfenidato para neuroaprimoramento farmacológico entre estudantesuniversitários. Einstein, São Paulo, v. I8, n. 4745, p. I-7, 2020. Disponível em: https://www.scielo.br/j/eins/a/Pz7jTFZmwxXtwfxKMGFSj9P/?format=pdf\&lang=pt.Ac e sso em: I4 nov. 2021.

CASTRO, C. X. L; LIMA, R. F. D. Consequências do transtorno do déficit de atenção e hiperatividade (TDAH) na idade adulta. Pepsic, São Paulo, v. 35, n. Io6, 6r-72, 2018. Disponível em:

http://pepsic.bvsalud.org/pdf/psicoped/v35nio6/o8.pdf. Acesso em: I2 nov. 202I. 
COGHILL, D. R. et al. Uma revisão sistemática da segurança do dimesilato de lisdexamfetamina. PubMed, [S.1.], v. 28, n. 6, p. 497-5II, jun./2014. Disponível em: https://www.ncbi.nlm.nih.gov/pmc/articles/PMC4057639/. Acesso em: I2 nov. 202I.

COMIRAN, Eloisa. Lisdexanfetamina: desenvolvimento e validação de métodos bioanalíticos por cromatografia líquida acoplada a detector de massas e avaliação farmacocinética preliminar. Lume, Porto Alegre, p. 19-225, out./2015. Disponível em: https://lume.ufrgs.br/handle/ror83/I48120. Acesso em: I set. 2021.

COSTA, Jessica Sophia. Metilfenidato: uso e quantificação. Repositório Institucional, [S.1.], p. I-42, mai./2or6. Disponível em: http://hdl.handle.net/ro284/5979. Acesso em: I2 set. 202I.

DOMITROVIC, Nathalia; CALIMAN, Luciana Vieira. As controvérsias sócio- históricas das práticas farmacológicas com o metilfenidato. Scielo, [S.1], v. 29, n. I63163, p. I-Io, jan./2017. Disponível em:

https://www.scielo.br/j/psoc/a/MHwXfV $7 K_{7}$ KYTFY6NDhdF $_{9} /$ /?lang=pt. Acesso em: II nov. 202I.

MONTEIRO, B. M. D. M. et al. Metilfenidato e melhoramento cognitivo em universitários: um estudo de revisão sistemática. Pepsic, [S.1.], v. 13, n. 4, p. 232-242, dez./2017. Disponível em: http://pepsic.bvsalud.org/pdf/smad/vi3n4/o8.pdf. Acesso em: 20 out. 2021.

NETA, B. R. B; NEVES, L. L. A; FIGUEREDO, M. L. R. D. S. A. S. Histórico de desenvolvimento e indicação terapêutica do Venvanse. FacUnicamps, [S.1], p. I- I8, ago./2020.

Disponível

em:

https://facunicamps.edu.br/cms/upload/repositorio_documentos/185_HIST\%CC3\%93R ICO $\% 20 D E \% 20 D E S E N V O L V I M E N T O \% 20 E \% 20 I N D I C A \% \mathrm{C}_{3} \% 87 \% \mathrm{C}_{3} \% 83 \mathrm{O} \% 20$ TE RAP\%C3\%8AUTICA\%2oDO\%20VENVANSE\%C2\%AE.pdf. Acesso em: ro nov. 202 I.

NETO, F. D. C. C. D. V. et al. O uso não prescrito de metilfenidato entre acadêmicos de medicina: uma revisão de literatura. Revista Interdisciplinar em Saúde, Cajazeiras, v. 5, n. 4, p. 759-773, set./2018. Disponível em: https://www.interdisciplinaremsaude.com.br/Volume_20/Trabalho_io.pdf. Acesso em: I4 nov. 202I.

OKAMURA, A. M. N. C. Análise comportamental e neuroquímica de ratos tratados com doses repetidas de lisdexanfetamina na infância e periadolescência. PPGF, Fortaleza, v. I65, p. I6-ıoo, fev./2019. Disponível em: http://www.repositorio.ufc.br/handle/riufc/4I47I. Acesso em: Io nov. 202I.

PIRES, M. D. S. et al. O uso de substâncias psicoestimulantes sem prescrição médica por estudantes universitários. Revista Científica Fagoc, $[\mathrm{S} .1]$, v. 3, n. 2, p. 22-29, jan./2018. Disponível

em: 
https://revista.unifagoc.edu.br/index.php/saude/article/view/370/347. Acesso em: 5 nov. 2021.

ROCHA, Bruna. Avaliação da frequência do uso do metilfenidato por estudantes de ensino superior. UNISC, Santa Cruz do Sul, p. I-63, jan./2or6. Disponível em: http://hdl.handle.net/ri624/I429. Acesso em: I set. 2021

SILVA, L. V. S. D. Farmacoterapia do transtorno do déficit de atenção. Repositório Instituicional, Florianópolis, p. I-45, dez./2020. Disponível em: https://repositorio.ufsc.br/handle/123456789/218567. Acesso em: 8 out. 2021

TAKEDA. Venvanse: Dimesilato de lisdexanfetamina. Takeda, [S.1], v. 55, n. 55, p. I-17, mar./202I. Disponível em: https://www.takeda.com/497830/siteassets/pt- br/home/whatwe-do/produtos/venvanse_bula-profissionais.pdf. Acesso em: 30 set. 202I.

VERGHESE, Corinne; ABDIJADID, Sara. Metilfenidato. PubMed, [S.1], v. 55, n. 55, p. I-Io, mai./202I. Disponível em: https://pubmed.ncbi.nlm.nih.gov/29494058/.Acesso em: II nov. 202I.

WACHHOLZ, Julia Haiana. Uso de estimulantes entre estudantes: análise de questionário online. Repositório PucGoias, Goiânia, p. I-30, out./2020. Disponível em: https://repositorio.pucgoias.edu.br/jspui/handle/I23456789/2098. Acesso em: roset. 2021. 